\title{
Caracterização isoenzimática de isolados humanos de Leishmania sp (Kinetoplastida: Trypanosomatidae) dos municípios de Rio Preto da Eva e Manaus, Estado do Amazonas
}

\author{
Isoenzymatic characterization of human isolates of Leishmania sp \\ (Kinetoplastida: Trypanosomatidae) from the municipalities \\ of Rio Preto da Eva and Manaus, State of Amazonas \\ Luanda de Paula Figueira ${ }^{1}$, Michele Zanotti ${ }^{1}$, Francimeire Gomes Pinheiro ${ }^{1}$ \\ e Antonia Maria Ramos Franco ${ }^{1}$
}

\begin{abstract}
RESUMO
Foram caracterizados/identificados por eletroforese de isoenzimas 23 isolados de Leishmania sp de pacientes dos municípios de Rio Preto da Eva e Manaus, analisando-se o grau de similaridade entre os organismos. Os resultados indicaram ocorrência de Leishmania guyanensis e Leishmania naiffi nestes dois ambientes e a heterogeneidade das amostras de Leishmania naiffi.
\end{abstract}

Palavras-chaves: Leishmania. Isoenzimas. Caracterização. Amazonas.

\begin{abstract}
Twenty-three isolates of Leishmania sp from patients in the municipalities of Rio Preto da Eva and Manaus were characterized and identified by means of isoenzyme electrophoresis and the degree of similarity between the organisms was analyzed. The results indicated that Leishmania guyanensis and Leishmania naiffi were present in these two environments and that the Leishmania naiffi samples were heterogenous.
\end{abstract}

Key-words: Leishmania. Isoenzymes. Characterization. Amazonas.

As leishmanioses compreendem uma gama de doenças encontradas ao redor do mundo em regiões tropicais e subtropicais. Suas diferentes formas clínicas constituem um grave problema de saúde pública ${ }^{15}$. A variedade de manifestações clínicas está associada a diferentes espécies do gênero Leishmania e à resposta imune do hospedeiro ${ }^{14}$. A identificação do parasita é essencial em qualquer estudo clínico patológico da doença, uma vez que há variação específica na sua virulência e resposta terapêutica. A caracterização e comparação de cepas de Leishmania isoladas de humanos, reservatórios e vetores são úteis no conhecimento ecológico e epidemiológico da infecção e controle da doença ${ }^{17}$. Pela dificuldade em se discriminar estes parasitas com critérios extrínsecos, têm-se utilizado diversos marcadores moleculares capazes de identificar e classificar os organismos de acordo com as características comuns ${ }^{7}$. A eletroforese de isoenzimas é um método considerado como padrão-ouro na caracterização de cepas de Leishmania ${ }^{3}$. Este estudo teve como objetivo caracterizar isolados de Leishmania sp de pacientes moradores dos municípios de Rio Preto da Eva e Manaus no Estado do Amazonas, e analisar o grau de similaridade entre os organismos isolados comparando com perfis de cepas de referência da Organização Mundial de Saúde, indicando assim as prováveis espécies circulantes nestes ambientes.

A Cidade de Rio Preto da Eva, criada na década de 60, servindo como conexão rodoviária entre Manaus e Itacoatiara, está localizada na orla de um rio com o mesmo nome da cidade, próxima ao Km 80 da Rodovia AM-010 (307’06“ S, $\left.59^{\circ} \mathrm{W}\right)$. Apresenta $5.591 \mathrm{~km}^{2}$ de área territorial. A população é formada por 24.858 pessoas, segundo o censo do Instituto Brasileiro de Geografia e Estatística realizado em 2007. 0 Município de Manaus, capital do Amazonas, situa-se à margem do Rio Negro. Tem uma área territorial de $11.401 \mathrm{~km}^{2}$. Em 2007, apresentou uma população estimada em cerca de 1.646.602 habitantes 9.

1. Laboratório de Leishmaniose e Doença de Chagas, Instituto Nacional de Pesquisas da Amazônia, Manaus, AM.

Apoio financeiro: INPA, FAPEAM

Endereço para correspondência: MSc Luanda de Paula Figueira. Laboratório de Leishmaniose e Doença de Chagas/CPCS-INPA. Av. André Araújo 2936, Aleixo, 69060-001 Manaus, AM.

Tel: 5592 3643-3068; Fax: 5592 3643-3061

e-mail: luandafigueira@yahoo.com.br

Recebido para publicação em 23/02/2008

Aceito em 27/08/2008 
Foram analisadas 23 amostras isoladas de lesões de pacientes provenientes de diferentes localidades dos municípios de Manaus e Rio Preto da Eva, AM, coletadas durante o período de 1997 a 2005 e criopreservadas em nitrogênio líquido no Laboratório de Leishmaniose e Doença de Chagas/CPCS/INPA. 0 estudo foi aprovado pelo Comitê de Ética em Pesquisa (CEP) com seres humanos do Instituto Nacional de Pesquisas da Amazônia (protocolo $\mathrm{n}^{\mathrm{0}}$ 027/2005). As amostras foram selecionadas aleatoriamente, sendo os estoques estabilizados in vitro e expandidos em meio líquido Schneider completo ${ }^{8}$, contendo $10 \%$ de soro fetal bovino inativado e antibióticos. Os isolados foram identificados pela técnica de eletroforese de isoenzimas em gel de agarose conforme protocolo previamente descrito ${ }^{3}$. Foram utilizados oito sistemas enzimáticos: malato desidrogenase (MDH, E.C.1.1.1.37), isocitrato desidrogenase (IDH, E.C.1.1.1.42) utilizando substrato com NAD e NADP, enzima málica (ME, E.C.1.1.1.40), glicose-6fosfato desidrogenase (G6PDH, E.C.1.1.1.49), 6-fosfogluconato desidrogenase (6PGDH, E.C.1.1.1.44), aconitato hidratase (ACON, E.C.4.2.1.3) e hexoquinase (HK, E.C.2.7.1.1). Os resultados obtidos com os marcadores foram analisados numericamente, utilizando-se o programa de computador NTSYS (versão 1.7, Exeter Software). Foi realizada análise fenética utilizando os coeficientes de similaridade de Jaccard, Simple Matching e Dice. 0 resultado final da análise numérica foi expresso em um dendrograma representando o relacionamento multivariado entre as OTU'S (Unidade Taxonômica Operacional $)^{2}$. Das amostras analisadas, verificou-se que 18 eram de Leishmania guyanensis e cinco de Leishmania naiffi, sendo 10 casos de Leishmania guyanensis e três de Leishmania naiffi, no município de Manaus, e oito de Leishmania guyanensis e dois de Leishmania naiffi, em Rio Preto da Eva, demonstrando a ocorrência destas espécies nestes municípios (Tabela 1). As lesões cutâneas de Leishmania guyanensis se apresentaram como úlceras únicas ou múltiplas, com ou sem crostas e as ocasionadas por Leishmania naiffi eram úlceras únicas de pequeno tamanho, papulosas ou pruriginosas, que evoluíam para lesão ulcerada.

Tabela 1- Origem e identificação das leishmanias isoladas de pacientes dos municípios de Rio Preto da Eva e Manaus, AM.

\begin{tabular}{lll}
\hline Espécies $^{\mathrm{a}}$ & \multicolumn{2}{c}{ Designação $^{\mathrm{b}}$ / Origem geográfica } \\
\hline Leishmania guyanensis & MHOM/BR/97/IM4365 & MHOM/BR/03/IM5079 \\
& MHOM/BR/99/IM4595 & MHOM/BR/04/IM5219 \\
& MHOM/BR/99/IM4641 & MHOM/BR/04/IM5221 \\
& MHOM/BR/00/IM4771 & MHOM/BR/04/IM5224 \\
& MHOM/BR/99/IM4961 & MHOM/BR/04/IM5227 \\
& MHOM/BR/03/IM5013 & MHOM/BR/04/IM5237 \\
& MHOM/BR/03/IM5023 & MHOM/BR/04/IM5238 \\
& MHOM/BR/03/IM5042 & MHOM/BR/05/IM5253 \\
& MHOM/BR/04/IM5225 & \\
MHOM/BR/05/IM5281 & \\
\hline Leishmania naiff & MHOM/BR/03/IM5012 & MHOM/BR/04/IM5232 \\
& MHOM/BR/03/IM5025 & MHOM/BR/05/IM5245 \\
& MHOM/BR/03/IM5039 & \\
\hline
\end{tabular}

a: identificação do estoque foi estabelecida através da análise de isoenzimas de acordo com o padrão isoenzimático e análise numérica ${ }^{23}$. b: designação: hospedeiros [M = Mammalia: HOM: Homo sapiens/país de origem/ano de isolamento/código de origem. BR: Brasil, AM: Amazonas].
A identificação das espécies foi obtida através da comparação dos perfis isoenzimáticos das amostras com as cepas de referência. As amostras analisadas por taxonomia numérica foram agrupadas em cinco zimodemas denominados INPA3, INPA4, INPA5, INPA6 e IOC-23. Este último foi nomeado de acordo com a classificação de Cupolillo ${ }^{3}$ por apresentar 18 isolados caracterizados como Leishmania guyanensis com perfil idêntico ao do IOC-23. As enzimas G6PDH e ME são consideradas como diagnósticas para Leishmania guyanensis e Leishmania panamensis. Como estas foram utilizadas neste estudo, descarta-se a possibilidade de algum destes isolados ser identificado como Leishmania panamensis. Para cada enzima, as bandas com mobilidade eletroforética idêntica foram consideradas como sendo o mesmo eletromorfo. Os oito loci estudados mostraram-se polimórficos para o subgênero Viannia. 0 número médio de alelos por locus foi de 4,8 (variação de 2 a 8 ) indicando a variabilidade genética do subgênero e o polimorfismo enzimático de Leishmania naiffi. Dentre os eletromorfos encontrados, alguns são considerados diagnósticos, ou seja, caracterizam determinados grupos ou zimodemas. Neste caso, a enzima G6PDH é um alelo diagnóstico que possibilita discriminar Leishmania naiffi, Leishmania guyanensis e Leishmania panamensis de outras espécies de leishmania ${ }^{3}$. 0 zimodema INPA-5 reuniu dois isolados de Leishmania naiffi de pacientes de Manaus (IM5012 e IM5025). Outros três zimodemas (INPA-3/IM5245, INPA-4/IM5039 e INPA-6/IM5232), desta mesma espécie, podem ser observados no fenograma (Figura 1) representados por isolados únicos. Em

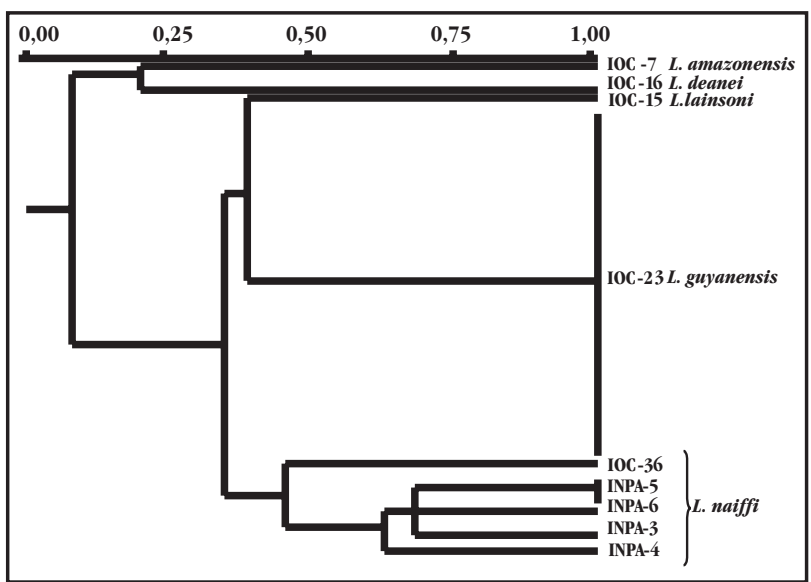

Figura 1 - Fenograma a partir do coeficiente de Jaccard, mostrando o grau de similaridade entre os diferentes zimodemas.

Rio Preto da Eva têm sido notificados diversos casos de leishmaniose, onde cerca de $80 \%$ são oriundos de diversos assentamentos ao longo da rodovia AM-010 ${ }^{12}$. Em 1998, Naiff descreveu a ocorrência de um caso humano diagnosticado como Leishmania naiffi no Km 93 da rodovia AM-010 de Rio Preto da Eva. Também foram notificados dois casos de infecção por Leishmania naiffi em humanos no município de Manaus. Quanto ao aspecto biológico, Naiff ${ }^{11}$ e Lainson \& Shaw ${ }^{10}$, referemse às raras lesões cutâneas produzidas na pele de hamster por Leishmania naiffi, fato também observado neste estudo, apesar do parasita poder ser reisolado em cultura até um ano após 
inoculação. Isto dificulta o diagnóstico da leishmaniose quando apenas o isolamento em hamster é realizado. É provável que Leishmania naiffi possa também produzir uma infecção benigna e oculta na pele humana e que a transmissão no Amazonas seja mais freqüente do que se tem observado.

Segundo a Fundação de Vigilância em Saúde ${ }^{5}$, Manaus é considerada como o município com maior número de casos da LTA. Naiff ${ }^{12}$, examinando um total de 108 pacientes oriundos do Estado do Amazonas, verificou que $86(77,4 \%)$ destes apresentavam a doença causada por Leishmania guyanensis. Romero e cols ${ }^{13}$, analisando a frequiência de infecção humana por Leishmania braziliensis em quatro dos municípios ao norte do Rio Amazonas (Manaus, Itacoatiara, Rio Preto da Eva e Presidente Figueiredo), demonstraram infecção por Leishmania guyanensis em 97\% (69/71) dos casos. A caracterização bioquímica e molecular de espécies dentro do gênero tem revelado alguns dos mecanismos envolvidos na heterogeneidade populacional de Leishmania. Os resultados de eletroforese de enzimas confirmam estudos anteriores ${ }^{3611}$ mostrando a aparente homogeneidade bioquímica dos isolados de Leishmania guyanensis oriundos de vários focos endêmicos na Amazônia. Um problema observado em Manaus, assim como em outros grandes centros, diz respeito ao local de origem da transmissão da doença. Freqüentemente, não se conseguem correlacionar as informações obtidas pelo paciente com os dados das análises laboratoriais/epidemiológicas, devido à incongruência destes. Em sua maioria, a transmissão da doença em moradores da zona urbana ocorre quando estes visitam sítios nas rodovias AM-010 (Manaus-Itacoatiara) e BR-174 (Manaus - Boa Vista) ou casas de parentes e amigos em áreas endêmicas de ambientes florestais; entretanto, nem sempre estes dados são informados. Além dessas duas espécies, casos de Leishmania amazonensis também foram descritos em Rio Preto da Evá.

\section{REFERÊNCIAS}

1. Castro EA, Luz E, Telles FQ, Pandey A, Biseto A, Dinsiski M, Sbalqueiro I, Soccol VT. Eco-epidemiological survey of Leishmania (Viannia) braziliensis
American cutaneous and mucocutaneous leishmaniasis in Ribeira Valley River, Paraná State, Brazil. Acta Tropica 93: 141-149, 2005.

2. Cupolillo E. Perfil enzimático e análise numérica de variantes do gênero Leishmania encontrados no novo mundo. Dissertação em Biologia Parasitária, Instituto Oswaldo Cruz, Fundação Instituto Oswaldo Cruz, p. 1992.

3. Cupolillo E, Grimaldi Jr G, Momen H. A general classification of the New Word Leishmania using numerical zymotaxonomy. The American Journal of Tropical Medicine and Hygiene 50:296-311, 1994.

4. Figueira LP, Pinheiro FG, Nogueira RW, Nery LCR, Mota KC, Franco AMR. Dinâmica da Leishmaniose Tegumentar no assentamento Iporá-AM, BR: II-Caracterização isoenzimática de isolados humanos humanos de Leishmania spp. (Kinetoplastida: Trypanosomatidae). Revista da Sociedade Brasileira de Medicina Tropical 41 (supl 1): 194, 2008.

5. Fundação Nacional de Saúde. Manual de Vigilância da Leishmaniose Tegumentar Americana. Ministério da Saúde, $2^{\text {a }}$ edição, Brasília, 2007.

6. Grimaldi Jr G, Momen H, Naiff RD, McMahon-Pratt D, Barrett T. Characterization and classification of leishmanial parasites from human, wild mammals and sand flies in the amazon region of Brazil. The American Journal of Tropical Medicine and Hygiene 44: 645-661, 1991.

7. Grimaldi Jr G, Tesh RB. Leishmaniasis of the New World: Current concepts and implications for future research. Clinical Microbiology Reviews 6: 230-250, 1993.

8. Hendricks LD, Wood DE, Hadjuk ME. Haemoflagellates: commercially available liquid media for rapid cultivation. Parasitology 76: 309-316, 1978.

9. Instituto Brasileiro de Geografia e Estatística: http:// www.ibge.gov.br. Acesso em: 13.02.08

10. Lainson R, Shaw JJ. New World leishmaniasis. Disponível em: http://www. topleyand Wilson.com/pdf/Parasitology20chapter.pdf. Acesso em: 10 de outubro de 2005.

11. Naiff MF. Leishmaniose Tegumentar na Amazônia. Distribuição geográfica dos agentes etiológicos na região. Dissertação. Mestrado em Biologia Celular e Molecular, Fundação Oswaldo Cruz, p. 1998.

12. Pereira MAS. Dinâmica da Leishmaniose Tegumentar Americana na área do Projeto de Assentamento Iporá, Rio Preto da Eva, AM. Manaus. Dissertação Mestrado em Entomologia, Instituto Nacional de Pesquisa da Amazônia, p. 2003.

13. Romero GAS, Guerra MVF, Paes MG, MAcêdo VO. Comparation of Cutaneous leishmaniasis due to Leishmania (Viannia) braziliensis and $L$. (V.) guyanensis in Brazil: therapeutic response to meglumine antimoniate. The American Journal of Tropical Medicine and Hygiene 65: 456-465, 2001.

14. Silveira FT, Lainson R, Corbett CEP. Clinical and Immunopathological Spectrum of American Cutaneous Leishmaniasis with Special Reference to the Disease in Amazonian Brazil. Memórias do Instituto Oswaldo Cruz 99: 239-251, 2004.

15. World Health Organization. Strategic Direction for Research. February, p.1-5, 2002 . 